\title{
Navigating Prostate Cancer: A Map of the Territory and Guidelines for Leveling the Playing Field Shared by Patients, Care Providers, Clinical Researchers, and Advocates
}

\author{
Al Pfadt ${ }^{1}$ and Joel Nowak ${ }^{2}$ \\ New York, USA \\ ${ }^{2}$ Weill Cornell Medicine, Us TOO New York and Cancer ABCs, New York, USA
}

${ }^{1}$ Former Research Scientist at the New York State Institute for Basic Research in Developmental Disabilities,

\section{*Corresponding author:}

\author{
Albert Pfadt \\ Former Research Scientist at the New York State \\ Institute for Basic Research in Developmental \\ Disabilities, New York, USA.
}

Received : August 28, 2019

Published : September 29, 2019

\begin{abstract}
Prostate cancer, like other complex biomedical conditions, must be understood from multiple perspectives. This article presents a formal analytic model to serve as a conceptual map for exploring 4 distinctly different domains of prostate cancer. A navigational metaphor is used to reflect the challenges one encounters in crossing over from one domain to the others, without explicit guidelines to aid in this endeavor. Such a model levels the playing field shared by patients, care providers, clinical researchers, and advocates so that informed decisions can be made regarding treatment. It also helps all sub-groups within the prostate cancer community to achieve a deeper understanding of this potentially lethal disease and communicate more effectively with each other.
\end{abstract}

affect his/her patient, who is assigned the role of passive participant, or simply as the object of treatment [2]. However, dissatisfaction with the traditional medical model extends far beyond insisting that patients should be recognized as partners in the decision-making process. There has been a rejection of the fundamental premise of the traditional medical model that disease entities can be reduced to their lowest common denominator. In the case of cancer, this reductionist mind-set has resulted in a focus on the structural, cellular characteristics of cancer and the complex genetic control mechanisms that transform a "normal cell" into a "cancerous process". For example, Vogelstein and Kinzler [3] flatly state that the essence of cancer can be summed up in a single sentence: 'it is a genetic disease'.

However, an alternative to this overly simplistic, reductionist model was proposed over 25 years by Engels [4]. Rather than reducing a medical condition to its most basic level, a biopsychosocial perspective considers it from increasing levels of complexity, including the conscious awareness of the patient responsibility for making the most important decisions that 
about his/her illness. From this perspective, a disease is not just something a patient "has". It can also be regarded as a process he/she helps to create and perpetuate. Instead of considering the patient as a passive victim of his/her condition, this new paradigm empowers the patient to assume authorship over a medical narrative that he/she co-creates and can change. Doctor and patient can be seen as engaging in a dialogue that uses a new language to transform the way that chronic illnesses like cancer are understood and treated. When viewed through the lens of this new medical model, cancer is not just a collection of signs and symptoms that can be observed using the latest imaging technology and represented in a patient's clinical history. Rather, it must be understood as something that affects the entire person and his/her social milieu. From a holistic perspective, cancer is now regarded as a chronic, multi-systemic disease that affects the person's body, mind, emotions, and spirit [5-7]. Furthermore, a patient's illness reverberates throughout his/her entire social network.

Hippocrates, regarded as the father of modern medicine, recognized this reality by reportedly saying it is more important to consider what person the disease has than it is to consider what disease the person has.' In this respect, he anticipated by about 2,000 years the development of a "new approach" to diagnosing and treating cancer that has been labeled "integrative oncology" [5]. Integrative oncology is an example of a healing-oriented medicine "that takes account of the whole person [mind, body, and spirit] as well as all aspects of lifestyle. It emphasizes the therapeutic relationship and makes use of all appropriate therapies, both conventional and complementary" [5,p.vii]. This perspective is also endorsed by Geffen [6] and Servan-Schreibner [7], as well in the plethora of self-help manuals echoing this theme [8-10]. When viewed from this holistic perspective, cancer can be thought of as existing in a patient's "life space", a term introduced by Lewin [11] to refer to the sum total of all factors affecting a person at a particular moment in time, as well as having a tangible reality as a bio-psychosocial process.

\section{A Formal Analytic Model as a Map for Navigating Prostate Cancer}

According to Rose and Dobson [12], a scientific model is fundamentally different from a theory in a number of ways. A theory is constructed by synthesizing information that has been verified by subjecting well-formulated hypotheses to falsification through a series of controlled experiments. A model, on the other hand, is a mental image or sometimes a physical representation of a phenomenon that interests a scientific community, created to capture its essential characteristics. A theory should be evaluated according to how useful it is in helping scientists predict and control the phenomena being investigated. Models, however, are evaluated according to different criteria. Since they are created to help the scientist understand the true nature of the subject matter being investigated, their success depends on how clearly that understanding can be conveyed to others. Accordingly, while theories are typically expressed in technical language with precise meanings that are often only understood by other scientists, models are formulated using familiar words and images that are readily understood by the general public.

In constructing their model of the visual cortex, Rose and Dobson [12] used three principles they recommended to others interested in creating similar models:

1. Attempt to identify and specify all aspects of the phenomenon being investigated so that others can understand its fundamental nature.

2. Simplify the representation of each of the model's parameters to 2 logical dichotomies, whenever possible.

3. Combine the most important dichotomies to create a framework for listing all of the logical possibilities.

In previous publications [13,14], I have described how to create a "formal analytic model" that provides a conceptual map for considering all dimensions that are associated with various clinical conditions (self-injurious behavior and Prader-Willi syndrome, for example) from a bio-psychosocial perspective. As Rose and Dobson [12] recommended, the basic dimensions of a formal analytic model are created by juxtaposing two mutually exclusive categories that are relevant to the phenomenon being considered. This results in a grid consisting of 4 quadrants or frames of reference. Taken together, they provide a conceptual map for exploring the illness in question so that it can be understood and treated in a comprehensive manner. Such a framework can be used to organize all the information that is currently known or potentially knowable about prostate cancer. As illustrated below, each quadrant provides a comprehensive way of thinking about prostate cancer from each of 4 distinctly different perspectives. Using this map helps to level the playing field shared by patients, care providers, clinical researchers, and advocates who are like blind men exploring an elephant. Each of these parties is considered to have valuable information to contribute if that information is shared openly and respectfully with the others. 


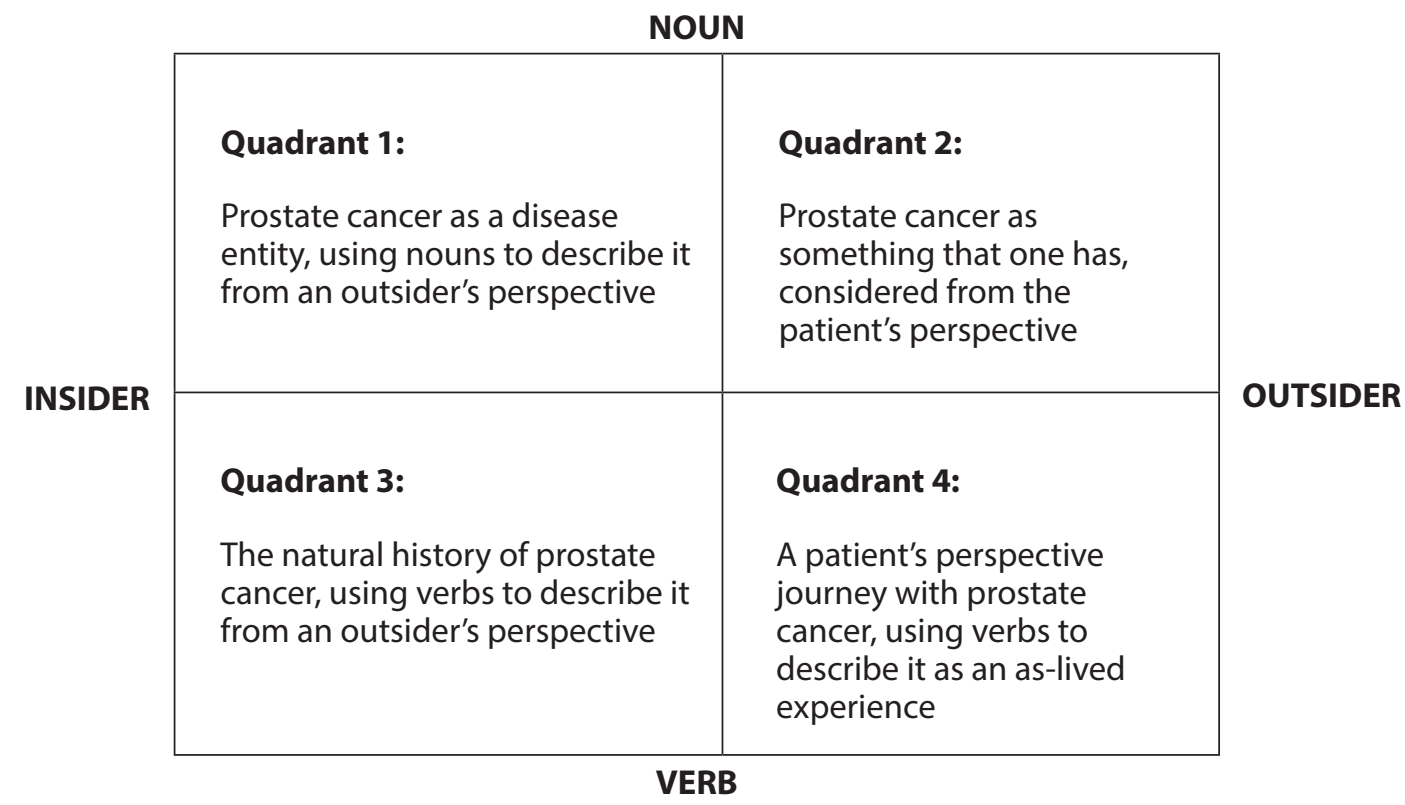

Figure 1: A formal analytic model of prostate cancer.

The upper half of the map, Quadrants 1 and 2 is formed by considering prostate cancer as a thing, using Nouns to describe its static properties. As an Object, it can be considered Either from the perspective of an observer [shown on the left] Or from the internal frame of reference of the man who has prostate cancer [shown on the right]. Traditionally, these polarities have been described as the Doctor's or Patient's perspectives. However, it is possible for a doctor to have prostate cancer and to describe his experience as a patient [15]. Likewise, a patient can adopt the detached perspective of a doctor when considering his/her prostate cancer [16]. However, it is not possible for one person to adopt both the insider and outsider perspectives at the same time. For example, when one looks at the reversible figure shown below in Figure 2, it is possible to see only a beautiful woman or an ugly hag in any instant, despite knowing that both perceptual experiences are possible.

The lower half of the map, Quadrants $\mathbf{3}$ and $\mathbf{4}$ is formed by using Verbs to describe the Natural History of Prostate Cancer as a dynamic process that takes place over time. Here again, the division into segments on the left or right depends on whether this process is described Either using the precise language of an Outsider that can be consensually validated Or subjectively, in terms of the as-lived experiences of the Insider who is taking this journey. Each frame of reference is explored below to illustrate how the model facilitates access to information on topics that might otherwise be considered to be restricted to a particular discipline or segment within the prostate cancer community.

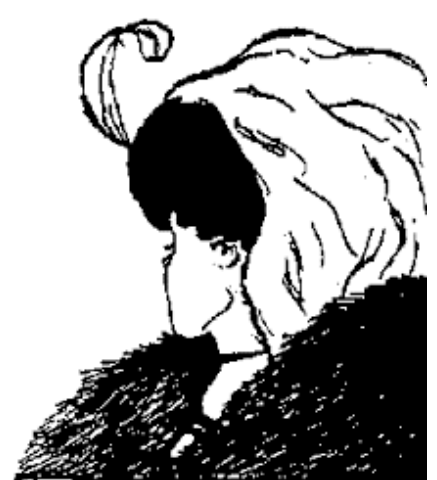

Figure 2: Reversible figure considered as an "ugly hag" (with a large nose buried in her shawl) or a "pretty woman" (wearing a flamboyant hat with a feather on top, viewed in profile).

\section{Quadrant 1 - Prostate Cancer as a Diagnostic Category - Considered from the Standpoint of an Outside Observer}

In the scientific literature, cancers are named according to a classification scheme that is based on their site of origin. Cancers are also classified based on information about the extent of their progression at the time of their diagnosis. Within this frame of reference, the primary objective is to use the map to consider issues related to diagnosing prostate cancer, determining its stage is at the time of diagnosis and making reasonable judgments about if and how it should be treated. Scholz [17] observed that prostate cancer is not a singular disease. He regards it as an umbrella term that covers five major stages and 15 sub-types. The literature referenced below helps provide some order to this classification process, while covering a wide range of topics of interest to patients, care providers, clinical researchers, and advocates.

1. Carcinoma of the Prostate $[\mathrm{CaP}]$ as a disease entity 
is explored in the classic handbook by Walsh and Worthington [18], now in its 4th edition.

2. Characteristics of a prostate cancer cell that enable it to metastasize can be described in terms of 8 hallmarks (essential features) and 2 enabling conditions [19].

3. Use of imaging studies and biomarkers to stage $\mathrm{CaP}$ is reviewed in Scholz [17], particularly chapters 3,4,5 and 6.

4. Perspectives on various treatment options are presented in $[16,18,20,21]$

\section{Quadrant 2 - Prostate Cancer as Something I Have - Becoming a Well-Adjusted Cancer Patient}

Being diagnosed with prostate cancer is like being air dropped into a familiar territory without navigational guidelines. For most men, hearing the words "you have prostate cancer" brings about a profound shift in our identity, even if we may have suspected for some time that this might be the case. This frame of reference provides a map for exploring the transition from "normalcy" into becoming a cancer patient, or as Sontag [1] put it, becoming a citizen in the kingdom of the sick. Roth [10] expanded upon the 5 stages of grief described by KublerRoss [22] to describe the terrain one must navigate in order to become a well-adjusted cancer patient.

1. Stop denying that a problem exists.

2. Overcome anger that one is a victim of unfair circumstances.

3. Resist the temptation to bargain for a reprieve from your cancer.

4. Correct any depression you might feel about the hopelessness of your situation.

5. Accept reality as it exists in the here and now.

This is not a linear process. Roth [10] correctly emphasized that patients zig-zag back and forth among various stages. However, they are guideposts that can be used to mark one's location on this first stage of the more challenging journey described in Quadrant 4.

Quadrant 3 - The Natural History of Prostate Cancer as a Biological Process - Considered from an Evolutionary Perspective

Nature is neither "good" nor "bad". Events unfold according to what can be described as "the natural scheme of things". At least this is the perspective taken when one looks at prostate cancer as a dynamic biological process using action verbs to describe it without the emotional overtones associated with the adjectives that are used to modify nouns as is the case in Quadrant 1, where cancers are described as "aggressive" or "potentially lethal". Here their growth patterns and characteristics are mapped out without any judgments about whether or not this is desirable or undesirable.

Hanin [23] presented a mathematical model of the natural history of prostate cancer based on certain biological hypotheses supported by the rapidly accumulating scientific literature on this topic. Other investigators [24,25] compare cancer's evolution in a patient's body to the origin of a new species from a common ancestral organism, using principles derived from Darwinian evolutionary theory. This evolution takes place in four stages or phases:

1. A period of Dormancy often lasts many years, during which time the primary tumor establishes a metastatic niche for subsequent colonization. This "seed and soil" hypothesis [26] accounts for the resistance many types of prostate cancer show to conventional attempts to "cure" them.

2. This is followed by a period of Emergence when the cancer is detected after having been dormant for many years, according to Horan [20, see pp. 67-72).

3. The tumor's Progression depends on the characteristics of the patient's cancer, its response to treatment, and what can broadly be described as the person's "lifestyle". Pfadt and Wheeler [27] presented a framework for graphically displaying this progression for an individual prostate cancer patient, in real time, as he responds to various treatments.

4. The Resolution stage of a particular patient's prostate cancer likewise depends on a variety of factors.

Most men don't require invasive treatments. They will die from other causes if they adhere to an appropriate active surveillance protocol [21]. Many others experience a durable remission, if not a cure, if their cancer is correctly diagnosed and treated [18]. Clinical trials with emerging treatment modalities offer hope for some patients who would otherwise have reached the end stages of conventional treatments [28]. Prostate cancer is typically a co-morbid disease. However, other medical conditions, as well treatment side-effects, can shorten a patient's 
lifespan [29].

\section{Quadrant 4 - The Journey through Prostate Cancer from the Time it is diagnosed until We Die with or from it - "Cancering" as Experienced by the Patient.}

The word "cancering" can be used to describe what takes place within this frame of reference. Most of the medical literature about prostate cancer has been written from the perspective of medical specialists, with the exception of a few extraordinary accounts provided by patients who describe their prostate cancer as a lived experience [15,30-32].

Geffen [6] categorized the navigational challenges encountered within this frame of reference in terms of 7 stages that provide landmarks for tracking progress during a patient's journey with prostate cancer. Each is described below, accompanied by references to consult for more details.

Stage 1 - Educate yourself about all aspects of cancer and cancer care by consulting resources that survey all of its dimensions [33]. A comprehensive resource specific to prostate cancer is the 4th Edition of Dr Patrick Walsh's guide to surviving prostate cancer [18].

Stage 2 - Connect with other prostate cancer patients using resources provided on the Internet (18, pp.503-505) to create a social network and find a support group,

Stage 3 - Learn to regard your body as a "garden" which requires tender loving care and constant attention so that you can thrive, not just survive, with prostate cancer [34,35].

Stage 4 - Begin the process of healing the trauma initiated by a diagnosis of prostate cancer and worsened by other adverse live events encountered during your journey. Roth [2015] described a type of "emotional judo" that helps one keep emotional balance and perspective during turbulent times.

Stage 5 - Learn to cope with the stressors you encounter by using mindfulness-based, stress reduction exercises [36].

Stage 6 - Identify your purpose, mission, and a vision of the legacy you wish to leave behind by conducting a thorough life assessment. A useful framework for discovering your authentic self during your journey with cancer is presented by Thomson [37]. The account by Ryan and Ryan [30] of their heroic battle with prostate cancer as a husband and wife team is as inspirational now as when it was published 40 years ago. They achieved their common purpose of completing their final novel during the cancer's terminal stages.
Stage 7 - A diagnosis of cancer does not mean that you have been given an "expiration date". Instead, it can kindle a process of spiritual growth [38-40].

As with any journey, there are many paths one can take along the way-some more clearly marked than others. In the case of cancer, as with life in general, they all lead to the same endpoint. Each of us will die due to some unforeseen circumstance. Receiving a diagnosis of cancer does not mean that we have been given an "expiration date". Rather, it is possible to consider this diagnosis and the ensuing experiences we have afterwards as providing a path to physical, mental, emotional, and spiritual recovery.

When confronted with the uncertainty that accompanies a diagnosis of cancer, we can either rebel against it or accept it for what it is. The Serenity Prayer has provided a source of guidance for many people who find themselves in this circumstance and ask for "Serenity to Accept what I cannot change, Courage to Change what I can, and Wisdom to Know the difference" [41]. A more secular version of that same advice was provided by Larson [42] in her meditation on John Cage, Zen Buddhism, and the inner life of artists: "Accept indeterminacy as a principle, and you see your life in a new light, as a series of seemingly unrelated jewel-like stories within a dazzling setting of change and transformation. Recognize you don't know where you stand, and you will begin to watch where you put your feet. That's when a path appears" [p. 20].

\section{SUMMARY}

A diagnosis of prostate cancer is often accompanied by complex physical, mental, emotional, and spiritual challenges that are difficult to navigate. Published literature that might be helpful in this regard is often written in technical language that is difficult for a layman to understand and is not typically available in one location. This article presents a formal analytic model to serve as a conceptual map, assisting men to complete what is often an arduous journey. It is also meant to help level the playing field shared by patients, care providers, clinical researchers, and advocates so that the results of their combined efforts are more accessible to all parties.

To that end; all of the references cited in this article, as well as additional resources, have been offered to a major cancer treatment center in New York City that provides services to men with prostate cancer in the larger metropolitan area. These could then be available to all interested members of 
the prostate cancer community who visit that location. If this offer is accepted, the article could function as a type of "Rosetta Stone" $[43,44]$ to assist these parties to collectively decipher information that might otherwise be difficult for any one individual to understand and use effectively. If adequate resources were made available, this information sharing model could be replicated in other locations.

\section{REFERENCES}

1. Sontag S (1977) Illness as metaphor. Farrar, Straus and Giroux, NY, USA.

2. Sessions RB (2012) The cancer experience: The doctor, the patient, the Journey. Rowman \& Littlefield Publishers Inc., Lanham, MD, USA, p. 205.

3. Vogelstein B, Kinzler K (2004) Cancer genes and the pathways they control. Nature Med 10(8): 789-999.

4. Engles G (1977) The need for a new medical model: A challenge for biomedicine. Science 196: 129-136.

5. Abrams D, Weil A (2009) Integrative oncology. Oxford University Press, NY, USA.

6. Geffen J (2006) The journey through cancer: Healing and transforming the whole person. Expanded and revised edition. Three Rivers Press, NY, USA.

7. Servan-Schreiber D (2011) Anticancer: A new way of life. ( $2^{\text {nd }}$ Ed.) Penguin Books, NY, USA.

8. Alschuler L, Gazella K (2011) Five to thrive: Your cutting edge cancer prevention plan. Active Interest Media Inc., El Segundo, CA, USA.

9. Thomson PAJ (2016) After-shock: From cancer diagnosis to healing: A step-by-step guide to help you navigate your way. Revised Ed. Roots \& Wings, New Paltz, NY, USA.

10. Roth AJ (2015) Managing prostate cancer: A guide to better living. Oxford University Press, NY, USA.

11. Lewin K (1951) Field theory in social science. Harper \& Brothers, NY, USA.

12. Rose D, Dobson V (1985) Models of the visual cortex. Wiley, NY, USA.

13. Pfadt A (1990) Diagnosing and treating psychopathology in clients with a dual-diagnosis. In Dosen A, Zwanikken G (Eds). Logon Publications, Leiden, Netherlands, p. 217224.
14. Pfadt A (1992) A map for exploring the behavioral phenotype of Prader-Willi syndrome. P-W Persp 1: 17-19.

15. DeVita A (2015) The death of cancer. Farrar, Straus and Giroux, NY, USA.

16. Sankey O (2015) Trouble with the man gland: Journeys of a scientist patient exploring the science of advanced prostate cancer. Schwartz Publishing House, Tempe, AZ, USA.

17. Scholz M (2015) The key to understanding prostate cancer. Self-published.

18. Walsh PC, Worthington JF (2018) Dr. Patrick Walsh's guide to surviving prostate cancer. $\left(4^{\text {th }} \mathrm{Ed}\right.$.). Grand Central Life \& Style, NY, USA.

19. Hanahan D, Weinberg R (2011) Hallmarks of cancer: The next generation. Cell, 144(5): 646-674.

20. Horan AL (2012) How to avoid the over-diagnosis and over-treatment of prostate cancer. Bloomfield, CO: On the Write Path Publishing Co., USA.

21. Klotz $L$ (2018) The science behind active surveillance. In Scholz M (Ed). The key to understanding prostate cancer. Self-published, p.55-67.

22. Kubler-Ross E (1969) On death and dying. Macmillan, NY, USA.

23. Hanin L (2011) Why victory in the war on cancer remains elusive: Biomedical hypotheses and mathematical models. Cancer 3(1): 340-367.

24. Lacina L, Coma M, Dvorankova B, Kodet O, Melegova N, Gal $P$ (2019) Evolution of cancer progression in the context of Darwinism. Antican Res 39(1): 1-16.

25. Willygard C (2016) Cancer: An evolving threat. Nature 532(7598): 166-168.

26. Fidler IJ (2003) The pathogensis of cancer metastasis: The "seed and soil" hypothesis revisited. Nat Rev Cancer 3(6): 453-458.

27. Pfadt A, Wheeler DJ (2019) Single subject studies in prostate cancer: How graphing PSA test results and testosterone levels using Process Behavior and Celeration Charts can provide insight and guide clinical decisions. Oncogen J 2(2): 10-18.

28. Norquist $L$ (2018) Cancer research: Striving to live longer and better. In Scholz M (Ed). The key to prostate cancer. 
Self-published, p. 355-362.

29. Turner J (2018) Health issues for men with prostate cancer. In Scholz M (Ed). The key to prostate cancer. Selfpublished, p. 371-377.

30. Broyard A (1992) Intoxicated by my illness. Clarkson Potter Publishers, NY, USA.

31. Korda M (1997) Man to man. Vintage Books, NY, USA.

32. Ryan C, Ryan CM (1979) A private battle. Simon and Schuster, NY, USA.

33. Wyatt $D$, Hulbert-Williams $N$ (Eds). Cancer and cancer care. Sage, NY, USA.

34. Espinosa G (2016) Thrive don't only survive! Dr. Geo's guide to living your best life before \&after prostate cancer. Riverdale Publisher, NY, USA.

35. Moyad M (2013) Promoting wellness for prostate cancer patients. Spry Publishing, Ann Arbor, MI, USA.

36. Kabat-Zinn J (2103) How to cope with stress, pain, and illness using mindfulness meditation. Bantam Books, NY,
USA.

37. Thomson PAJ (1979) Track your truth: Discover your authentic self. Roots \& Wings, New Paltz, NY, USA.

38. Ryan C, Ryan CM (1979) A private battle. Simon and Schuster, NY, USA.

39. Kubler-Ross E, Kessler D (2000) Life lessons. Scribner, NY, USA.

40. Kurtz E, Ketcham K (2014) Experiencing spirituality. Penguin, NY, USA.

41. Servan-Schreibner D (2011) Not the last goodbye: On life, death, healing, and cancer. Viking, NY, USA.

42. The Serenity Prayer is attributed to Reinhold Niebuhr, who is reported to have used it in unpublished sermons as early as 1933 .

43. Larson K (2013) Where the heart beats. Penguin, NY, USA.

44. Dalby A, et al. (2019) Rosetta Stone. Wiki J of Humanities, 2(1): 1 